\title{
A Model Calculation of Temperature and Size Dependent Thermoluminescence Intensity from Silicon Nanostructures
}

\author{
Nebiyu Gemechu Debelo \\ Department of Physics, Mizan-Tepi University, \\ College of Natural and Computational Science, Tepi, Ethiopia \\ e-mail: nafsif@gmail.com
}

Received 04 August 2011, Accepted 10 October 2011

\begin{abstract}
Thermoluminescence (TL) from silicon nanostructures has received considerable attention in recent years. Despite of some theoretical and experimental investigation, the origin and mechanism of TL is not clearly understood. Most of the bulk materials start showing fascinating behaviors as their size is reduced to nano level. The ability to emit light as a consequence of absorption of energy (luminescence) is one of these exotic behaviors. TL is the thermally stimulated emission of light due to absorption of energy from radiation. So, there is a direct relationship between temperature (due to absorption of heat or radiation) and the TL intensity. Starting from the concept of bulk material, we derive the mathematical expression for the temperature and size dependent TL intensity using model calculation. We specifically calculate the TL intensity as a function of temperature and nanoparticle size.
\end{abstract}

Keywords: Thermoluminescence; Trapping; Detrapping; Rrecombination; Quantum confinement.

\section{Introduction}

Thermoluminescence is the emission of light from an insulator or semiconductor when it is heated, that is the thermally stimulated emission of light following the previous absorption of energy from radiation ${ }^{1,2)}$. There are three essential ingredients necessary for the production of thermoluminescence ${ }^{1)}$. Firstly, the material must be an insulator or semiconductor-metals which do not exhibit luminescent properties. Secondly, the material must have at some time absorbed energy during exposure to radiation. Thirdly, the luminescence emission is triggered by heating the material. In addition, there is one important property of thermoluminescence. It is a particular characteristic of thermoluminescence, namely: once heated to excite the light emission, the material can not be made to emit thermoluminescence again by simply cooling the specimen and reheating. In order to re-exhibit the luminescence, the material has to be re-exposed to radiation, whereupon raising the temperature will once again produce light emission $^{1)}$. The fundamental principles which govern the production of thermoluminescence are essentially the same as those which govern all luminescence processes, and in this way the thermoluminescence is one of a large family of luminescence phenomena. Luminescence can be produced because of two cases. One is due to the recombination of free electrons with trapped holes and the other is due to the recombination of free holes with trapped electrons. But in our model we have worked for the first case i.e. luminescence due to the recombination of free electrons with trapped holes.

\section{Model Calculation}

We know that the electronic current density from the surface of bulk solid (i.e. number of electrons per unit area of the solid) is given by the following equation.

$$
J=-\frac{e m_{e} K_{B}^{2} T^{2}}{2 \pi^{2} \hbar^{3}} \exp \left(-\frac{E_{0}}{K_{B} T}\right)
$$

Where $E_{0}$ is the minimum amount of energy required to make an electron free from the interior of the solid $^{3)}$. For nanostructured semiconductor materials (quantum dots, wires or wells) $E_{0}$ correspond to the band gap energy, $E_{g}$. Let us now consider a particular situation of detrapping the electrons at trap center by applying thermal energy. Here the minimum amount of energy required for detrapping is equal to the trap depth $E$. Therefore the current density due to detrapped electrons is given by Equation (2) below.

$$
J=-\frac{e m_{e} K_{B}^{2} T^{2}}{2 \pi^{2} \hbar^{3}} \exp \left(-\frac{E}{K_{B} T}\right)
$$

Since thermoluminescence is due to the annihilation of detrapped (free) electrons with trapped holes at trap center, the thermoluminescence intensity $I_{t h}$ is directly proportional to the recombination rate. But the recombination rate is directly proportional to the number $n$ of detrapped electrons; hence $I_{t h}$ is directly proportional to the number of free electrons $n$. Hence,

$$
I_{t h} \sim n
$$

But $n$ can be expressed as $n=A J$, where $J$ is the current density due to detrapped electrons and $A$ is the area, which is constant for a given sample. Therefore,

$$
I_{t h} \sim A J
$$

Another important idea is that the detrapped electrons may follow two path ways (radiative and non-radiative path ways). But it is electron that follow radiative path way that contribute to the thermoluminescence 
intensity, so that we can conclude that is also directly proportional to the rate of radiative recombination $R_{\text {rad. }}$

$$
I_{\text {th }} \sim R_{\text {rad }} A J
$$

Once more $I_{t h}$ is directly proportional to the probability per unit time $p$ of the release of an electron from the trap.

$$
I_{\text {th }} \sim p R_{\text {rad }} A J
$$

But $p=s \exp \left(-\frac{E}{K_{B} T}\right)$, where $\mathrm{s}$ is the trapping parameter [1]. For quantum dot (approximately spherical) of well defined size distribution, $A=4 \pi \mathrm{r}^{2}$, where $r$ is the radius of the dot. But it is found that $s$ varies with temperature like $T^{-b}$, where $-2<\mathrm{b}<2$ [1]. Using (2), (3), (4), (5) and (6), the thermoluminescence intensity $I_{t h}$ can be formulated as in Equation (7).

$$
I_{\text {th }}=C_{1} R_{\text {rad }} d^{2} \exp \left(\frac{-2 E}{K_{B} T}\right)
$$

where $C_{1}$ is constant and $\mathrm{d}$ is the diameter of the sample.
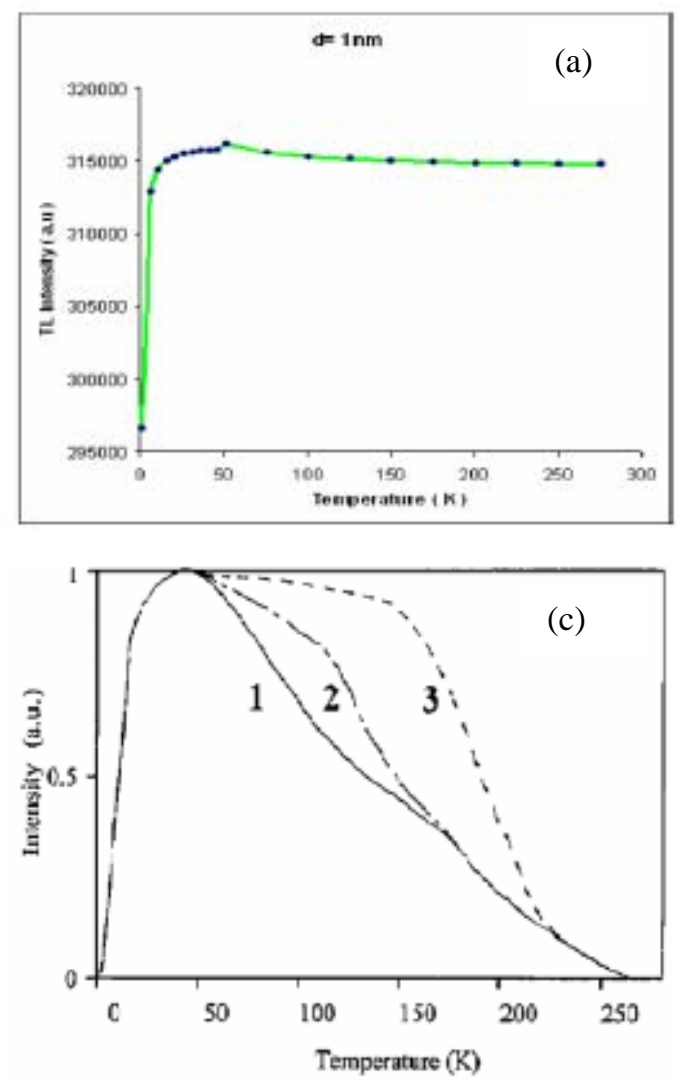

Equation (7) gives the thermoluminescence intensity as function of temperature and size of the dot.

\section{Results and Discussion}

\subsection{TL intensity versus temperature}

Starting from the concept of bulk material we have applied very crucial concepts and have gone through logical steps to reach at the final expression for the temperature dependent thermoluminescence intensity. For a fixed value of $b$ (which is approximately equal to 1.99) the thermoluminescence intensity has a peak value at a temperature of $50 \mathrm{~K}$ and starts decreasing after this temperature. We have worked for $1-300 \mathrm{~K}$ temperature range and found out that our result is in good agreement with the experimental results ${ }^{4)}$. As it can be seen from the Figure 1: (a),(b) and (c), the TL intensity increases with temperature and attains its maximum value near $50 \mathrm{~K}$ (with sharp peaks) and slowly decreases after this temperature. However, as compared to the experimental result (d), the TL intensity obtained from this model decays much slower after a temperature of $50 \mathrm{~K}$ and this may be due to the simplicity of our model.
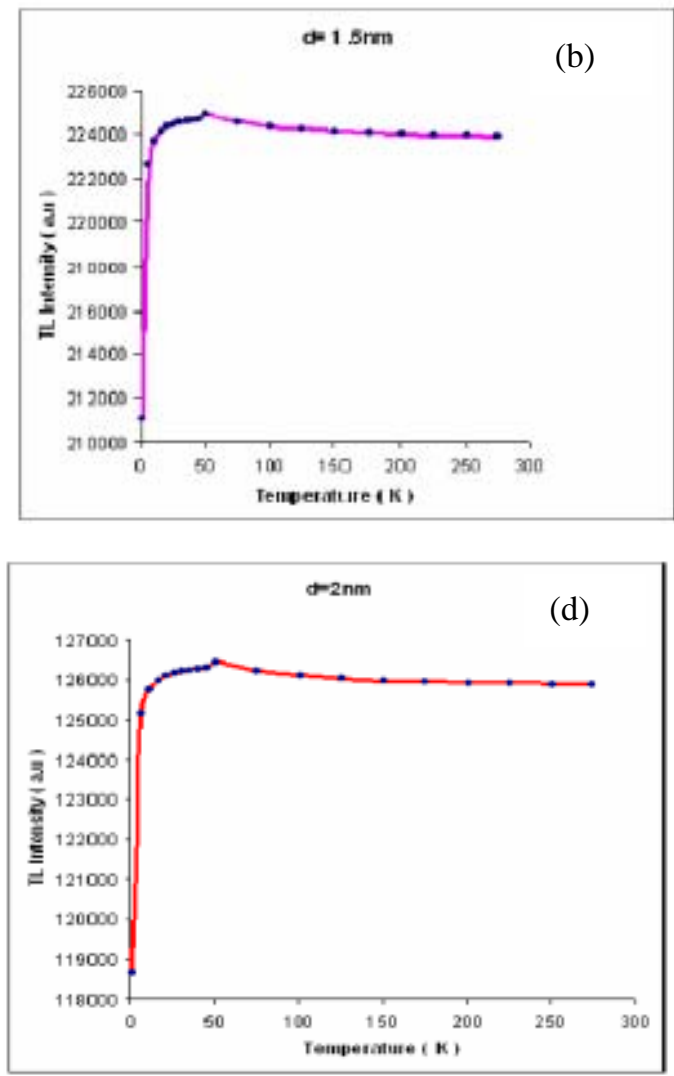

Figure 1. The calculated TL intensity for 1nm size (a), 1.5nm size (b), 2nm size (c) and the experimental plot (d). 
This theoretical model is also in good agreement with the experimental results for temperatures lower than $1 \mathrm{~K}$. For such lower temperatures, the exponential term approaches zero and hence the TL intensity drops to zero ${ }^{4)}$.

Therefore, this model has shown that applying the concept of bulk material is sometimes very important to understand the new behaviors reflected by materials at nanolevel. We can say that this model is a powerful approach for calculating the temperature and size dependent TL intensity. Of course the model has some limitations. We could not observe the fast decay mechanism of the TL intensity after $50 \mathrm{~K}$ as the experimental result.

\subsection{TL intensity versus size}

We are also interested in calculating the TL intensity as a function of the size of the dot for a given temperature. Figure 2 shows the calculated TL intensity versus size at different temperatures As expected, we observed that for a given temperature, the TL intensity increases with decreasing the size.

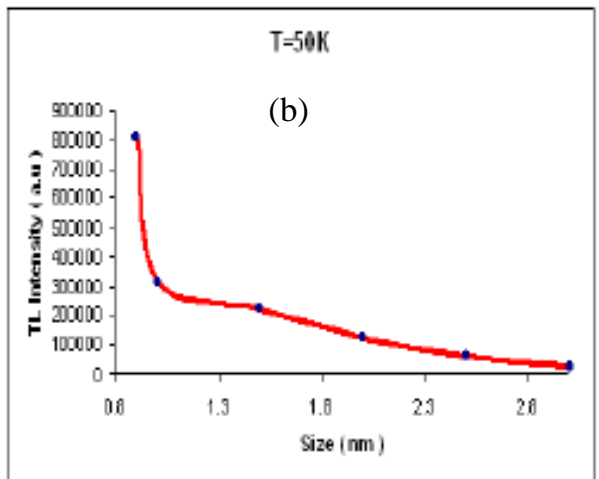

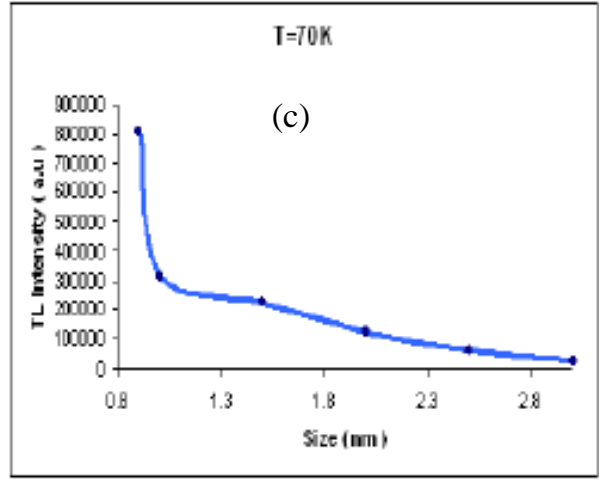

Figure 2. The calculated TL intensity versus size at different temperatures.

This is also due to the quantum confinement effect which allows direct band to band radiative recombination following the widening of band gap. From this result one can easily understand that the TL intensity increases with increasing the band gap of the dot; because the reduction in size allows the increment of band gap.

\section{Conclusion}

In this work we have developed a model for calculating the TL intensity as a function of temperature and size of the nanoparticle. We tried to include some concepts from bulk material and obtained the expression for the TL intensity from the dot with some possible modifications.

Some of our results are quite new and can also be applied to $\mathrm{Ge}$, GaAs, or other nanoparticles. In general we have observed that the temperature dependence of the TL intensity is much similar to that of the PL and EL intensities. This shows that the mechanism of luminescence in the three types of luminescence is in general similar to one another.

\section{References}

1. S.W.S., Mckeever, Thermoluminescence of Solids, Cambridge Solid State Science Series, Oklahoma State University, 396, 1988.

2. U. Pal et al., Thermoluminescence properties of $\mathrm{ZnO}$ and $\mathrm{ZnO}: \mathrm{Yb}$ nanophosphors, Appl. Phys. Lett., 89, 1-3, 2006.

3. K. Charles, An Introduction to Solid State Physics, $7^{\text {th }}$ Edition, University of California, Berkeley, 2005.

4. Y. A. Skryshevskii. and V. A. Skryshevskii, Thermally Simulated Luminescence, J. Appl. Phys. 89, 2711, 2001. 